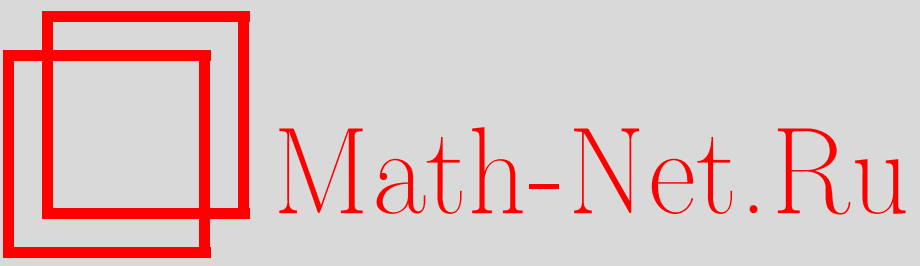

С. Босколо, С. К. Турицын, В. Ю. Новокшенов, Й. Нийхоф, Самоподобные параболические оптические уединенные волны, ТМФ, 2002, том 133, номер 3, 386397

DOI: https://doi.org/10.4213/tmf405

Использование Общероссийского математического портала Math-Net.Ru подразумевает, что вы прочитали и согласны с пользовательским соглашением

http://www.mathnet.ru/rus/agreement

Параметры загрузки:

IP: 54.210 .77 .194

26 апреля 2023 г., 17:50:52 
ТЕОРЕТИЧЕСКАЯ

И МАТЕМАТИЧЕСКАЯ

ФИЗИКА

Том 133, № 3

декабрь, 2002

(C) 2002 г.

С. Босколо*, С.К. Турицын*, В.Ю. Новокшенов ${ }^{\dagger}$, Й. Х.Б. Нийхоф

\section{САМОПОДОБНЫЕ ПАРАБОЛИЧЕСКИЕ ОПТИЧЕСКИЕ УЕДИНЕННЫЕ ВОЛНЫ}

Исследованы решения нелинейного уравнения Шредингера (НУШ) с усилением, которое описывает оптический импульс, распространяющийся в усиливающей среде. Построено квазиклассическое самоподобное решение с параболической временно́й вариацией, соответствующее энергосодержащему ядру асимптотически распространяющегося импульса в усиливающей среде. Самоподобное ядро сшивается с помощью функций Пенлеве с решением линеаризованого уравнения, которое соответствует низкоамплитудным хвостам импульса. Оказывается, что аналитическое решение в точности воспроизводит численно полученное решение НУШ.

Ключевые слова: нелинейная оптика, самоподобие, генерация параболических импульсов.

\section{1. ВВЕДЕНИЕ}

Самоподобие является фундаментальным физическим свойством, которое широко исследовалось во многих областях физики [1]. В оптике методы самоподобия были недавно применены к изучению распространения импульса в волоконных усилителях с нормальной дисперсией. Было показано, что параболические импульсы с квадратичной по времени фазой являются приближенно самоподобными решениями нелинейного уравнения Шредингера (НУШ) с усилением в пределе высокой интенсивности [2]. Эти результаты были подтверждены экспериментально в работе [3] и обобшили предыдушие теоретические исследования по распространению параболических импульсов в оптических волокнах [4].

В работах [2] и [4] вывод решений параболической формы основан на допушении о том, что линейным дисперсионным членом в уравнении для амплитуды поля по сравнению $c$ нелинейным членом можно пренебречь аналогично квазиклассическому приближению в квантовой механике. Хотя центральное ядро решения уже было описано в литературе для некоторых физических задач [2], [4], исчерпывающей теории пока нет. Настоящая

${ }^{*}$ Photonics Research Group, School of Engineering and Applied Science, Aston University, Birmingham, UK

${ }^{\dagger}$ Институт математики Уфимского научного центра РАН, Уфа, Россия

${ }^{\ddagger}$ Marconi Solstis, Stratford-Upon-Avon, UK 
работа посвящена подробному математическому анализу решений НУШ с усилением. Отправной точкой является анализ эволюции параметра, определяемого как отношение линейного члена к нелинейному, в плоскости время-расстояние. Это позволяет выделить три области на временно́й оси, которые соответствуют ядру, хвостам и узкой зоне перехода во временно́м профиле импульса, асимптотически распространяющегося в усилителе. НУШ далее решается в каждой из этих областей. Во внутренней области для нахождения самоподобного решения с параболическим временны́м профилем применяется квазиклассическое приближение. Во внешней области мы решаем линеаризованое НУШ. Квазиклассическое параболическое решение в центральной части при этом сшивается с линейным в небольшой промежуточной переходной области. Следуя работе [5], уравнение для амплитуды поля в переходной области можно редуцировать ко второму уравнению Пенлеве (РІІ). Асимптотическое поведение трансцендентов Пенлеве и задачи связи для уравнений Пенлеве были рассмотрены во многих работах (см., например, [6], [7] и приведенную в них библиографию). В данной работе, используя асимптотические результаты для РІІ, мы решаем задачу сшивки амплитуд. Построение решений завершается сшивкой фазы поля. Наш анализ дает обоснование сделанного в работах [2] и [4] интуитивного предположения о том, что параболическая аппроксимация формы импульса справедлива в центральной части асимптотического импульса. Более того, это дает полное описание формы и основных свойств импульсов высокой мошности, порожденных в усиливаюшей среде.

Работа построена следуюшим образом. В начале мы представляем основную модель, управляющую распространяющимся в усиливающей среде оптическим импульсом. В разделе 2 мы даем обзор вывода самоподобных параболических решений НУШ в пределе высокой интенсивности и сравниваем эти решения с результатами численного моделирования. В разделе 3 мы формулируем и аналитически решаем задачу сшивки. Показано, что построенные таким способом решения точно воспроизводят свойства импульса, наблюдаемые при численном моделировании. В заключительном разделе приводятся полученные результаты.

\section{2. ОСНОВНЫЕ УРАВНЕНИЯ}

Рассмотрим эволюцию локализованного оптического импульса в усиливающей среде в отсутствие насышения усиления для падающих импульсов с меньшей спектральной шириной линий, чем ширина линии усиления. В этом случае распространение импульса можно описывать при помоши НУШ с усиливающим членом [8]

$$
i \psi_{z}-\frac{\beta_{2}}{2} \psi_{t t}+\sigma|\psi|^{2} \psi=i \frac{g(z)}{2} \psi
$$

где $\psi(z, t)$ - медленно изменяющаяся огибающая импульса в движущейся вместе с импульсом системе координат, $\beta_{2}$ и $\sigma$ - параметры соответственно дисперсии групповой скорости и нелинейности, а $g(z)$ - профиль усиления вдоль волокна. Мы ищем решение уравнения (1) в виде

$$
\psi(z, t)=a(z) F(\eta, \xi) e^{i C(z) t^{2}}
$$


где введены новые самоподобные переменные

$$
\xi=\frac{t}{\tau(z)}, \quad \frac{d \eta}{d z}=\sigma a^{2}(z)
$$

Здесь $a(z)$ имеет ту же размерностость, что и $\psi(z, t)$, и описывает эволюцию пика амплитуды импульса с изменением расстояния, $\tau(z)$ - характеристическая ширина, $C(z)$ параметр нелинейного изменения частоты, а $F(\eta, \xi)$ - нормированая безразмерная функция, которая описьвает эволюцию временно́го профиля через его $\xi$-зависимость; мы также допускаем зависимость от $\eta$ для учета вкладов в пик амплитуды и фазу. Преобразования (2) и (3) дают связанные уравнения для $a, \tau, C$, и $F$ :

$$
\begin{gathered}
\frac{a_{z}}{a}=\beta_{2} C+\frac{g}{2} \\
\frac{\tau_{z}}{\tau}=-2 \beta_{2} C \\
\left(C_{z}-2 \beta_{2} C^{2}\right) \tau^{2}=-\lambda \sigma a^{2}, \\
i F_{\eta}+\left(|F|^{2}+\lambda \xi^{2}\right) F-\frac{1}{c(\eta)} F_{\xi \xi}=0, \quad c=\frac{2 \sigma a^{2} \tau^{2}}{\beta_{2}}
\end{gathered}
$$

где $\lambda$ - произвольный параметр, связанньй с мощностью импульса. Уравнения (4)-(6) можно переписать таким образом, чтобы получить соотношение между $a$ и $\tau$ :

$$
a^{2}(z) \tau(z)=a^{2}\left(z_{0}\right) \tau\left(z_{0}\right) \exp \int_{z_{0}}^{z} d z^{\prime} g\left(z^{\prime}\right)
$$

а также записать уравнение второго порядка для $\tau$

$$
\tau_{z z}=\frac{2 \beta_{2} \sigma \lambda a^{2}}{\tau}
$$

с дополнительным граничным условием $\left(\tau_{z}\right)_{z=z_{0}}=-2 \beta_{2} C\left(z_{0}\right) \tau\left(z_{0}\right)$. Здесь $z_{0}-$ произвольная начальная точка.

Разделяя функцию $F$ на вешественную амплитуду $A$ и фазу $\Phi$,

$$
F(\eta, \xi)=A(\eta, \xi) e^{i \Phi(\eta, \xi)}
$$

из уравнения (7) получаем “гидродинамическую” систему уравнений:

$$
\begin{gathered}
\left(A^{2}\right)_{\eta}-\frac{2}{c(\eta)}\left(A^{2} \Phi_{\xi}\right)_{\xi}=0 \\
\Phi_{\eta}+\frac{1}{c(\eta)}\left(\frac{A_{\xi \xi}}{A}-\left(\Phi_{\xi}\right)^{2}\right)-\left(A^{2}+\lambda \xi^{2}\right)=0 .
\end{gathered}
$$




\section{3. ПАРАБОЛИЧЕСКИЕ ИМПУЛЬСЫ ВЫСОКОЙ ИНТЕНСИВНОСТИ}

Рассмотрим сначала возможность приближенного описания энергосодержащего ядра. Определим параметр $\epsilon$ как отношение члена с линейной дисперсией к нелинейному члену в уравнении (11б):

$$
\epsilon(\eta, \xi)=\left|\frac{A_{\xi \xi}}{c(\eta) A^{3}}\right| .
$$

В этом разделе мы сконцентрируемся на свойствах распространения импульсов высокой интенсивности или, другими словами, на решениях, удовлетворяющих условию

$$
\epsilon \ll 1,
$$

которое соответствует так называемому квазиклассическому пределу уравнения (1). Когда оно выполнено, можно пренебречь членом, соответствуюшим линейной дисперсии в уравнении (11б), и получающаяся тогда система уравнений для $A$ и $\Phi$ принимает вид

$$
\begin{gathered}
\left(A^{2}\right)_{\eta}-\frac{2}{c(\eta)}\left(A^{2} \Phi_{\xi}\right)_{\xi}=0, \\
\Phi_{\eta}-\frac{1}{c(\eta)}\left(\Phi_{\xi}\right)^{2}-\left(A^{2}+\lambda \xi^{2}\right)=0 .
\end{gathered}
$$

Мы ишем решение уравнений (14), имеюшее самоподобную форму, т.е. $A=A(\xi)$. Более того, если мы полагаем $\Phi_{\xi}=0$, то легко найти решение с параболическим распределением интенсивности

$$
\begin{gathered}
A(\xi)=\left[\lambda\left(1-\xi^{2}\right)\right]^{1 / 2}, \quad|\xi| \leqslant 1, \\
\Phi(\eta)=\lambda \eta,
\end{gathered}
$$

которое приводит к следующей зависимости энергии импульса от его параметров:

$$
U=\int_{-\infty}^{+\infty} d t|\psi|^{2}=\frac{4}{3} \lambda a^{2} \tau .
$$

Теперь можно обобшить уравнения, моделируюшие динамическую эволюцию решения уравнения (1) как импульс высокой интенсивности. Из вида члена, соответствуюшего амплитуде:

$$
\begin{aligned}
& |\psi(z, t)|=\left(\frac{3 U(z)}{4 \tau(z)}\right)^{1 / 2}\left[1-\left(\frac{t}{\tau(z)}\right)^{2}\right]^{1 / 2}, \quad|t| \leqslant \tau(z), \\
& |\psi(z, t)|=0, \quad|t|>\tau(z),
\end{aligned}
$$

ясно, что решение соответствует импульсу с параболическим профилем интенсивности, причем точка пересечения с нулем $\tau(z)$ определяет эффективную ширину импульса. В фазовом члене

$$
\arg \psi(z, t)=\lambda \eta(z)+C(z) t^{2}
$$


решения $\eta(z)$ и $C(z)$ имеют вид

$$
\begin{gathered}
\eta(z)=\eta\left(z_{0}\right)+\left(\frac{3 \sigma}{4 \lambda}\right) \int_{z_{0}}^{z} d z^{\prime} \frac{U\left(z^{\prime}\right)}{\tau\left(z^{\prime}\right)}, \\
C(z)=-\left(2 \beta_{2}\right)^{-1}\left(\frac{d}{d z}\right) \ln \tau(z) .
\end{gathered}
$$

В уравнениях (17)-(20) скейлинг амплитуды и ширины импульса из их начальных значений можно вычислить, исходя из уравнений

$$
\begin{gathered}
U(z)=U\left(z_{0}\right) \exp \int_{z_{0}}^{z} d z^{\prime} g\left(z^{\prime}\right), \\
\frac{d^{2} \tau}{d z^{2}}=\frac{3 \beta_{2} \sigma U(z)}{2 \tau^{2}} .
\end{gathered}
$$

Отметим, что вывод такого решения основан на допушении (13). Эту аппроксимацию можно обосновать апостериори для параболического импульса, задаваемого уравнениями (17)-(20), поскольку $\epsilon \approx 2 \beta_{2} / 3 \sigma \tau U \ll 1$ за исключением области вблизи краев импульса.

Теоретические предсказания, следуюшие из уравнений (17)-(22), сравнивались с результатами численного моделирования уравнения (1). Мы моделировали распространение гауссова импульса полной ширины при полуширине импульса 0.5 пс с энергией 70 пДж в волоконном усилителе длиной $4 \mathrm{~m}$, интегрированным усилением 25 дБ, $\sigma=$ $6 \times 10^{-3} \mathrm{Bт}^{-1} \mathrm{M}^{-1}$ и $\beta_{2}=35 \times 10^{-3}$ пс $^{2} \mathrm{M}^{-1}$ [3]. Поскольку определяемое уравнениями (17)-(20) решение находится для любого профиля усиления $g(z)$, причем функциональный вид $g(z)$ определяет только самоподобный скейлинг распространяюшегося в усилителе импульса (см. уравнения (21) и (22)), без потери обшности во всей статье мы сосредоточимся на случае постоянного профиля усиления $g(z)=g_{a}$ при $g_{a}=1.44 \mathrm{M}^{-1}$.
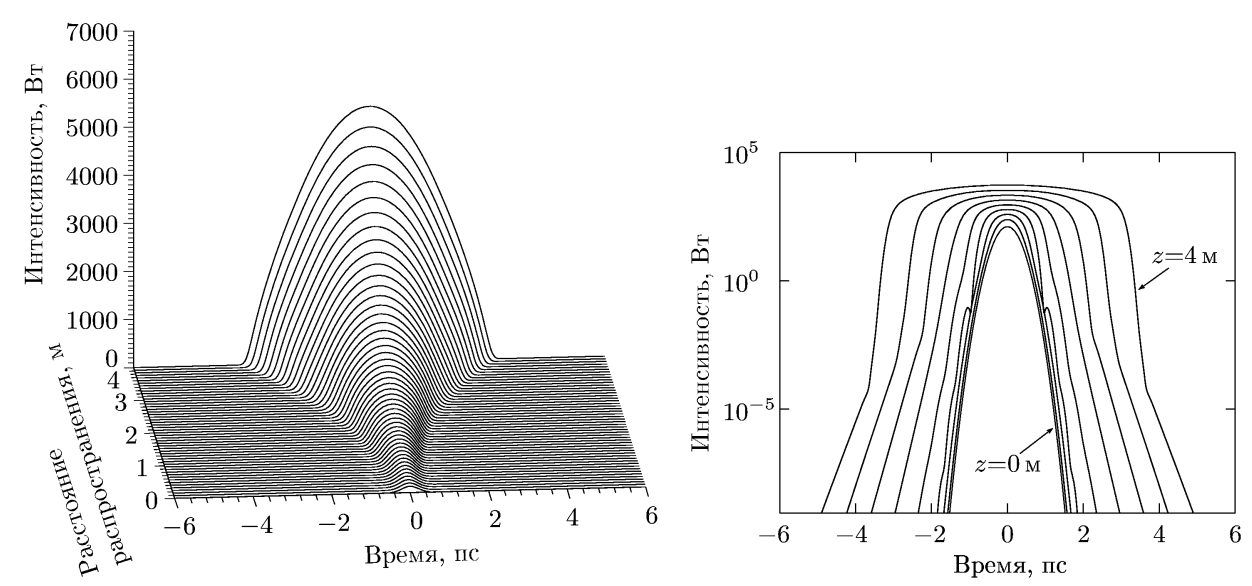

Рис. 1. Эволюция импульса в усилителе. В левой части в линейном масштабе показана эволюция профиля интенсивности, а в правой профили интенсивности нанесены в логарифмическом масштабе с шагом $0.5 \mathrm{~m}$. 
На рис. 1 показана эволюция импульса, полученная при моделировании НУШ, показывающая эволюцию профиля интенсивности с изменением расстояния распространения. Можно видеть, что когда падающий гауссов импульс усилен до высокой интенсивности, он эволюционирует к параболическому импульсу во второй половине усилителя. Интересное свойство, видное из графика в логарифмическом масштабе, состоит в том, что асимптотический импульс демонстрирует самоподобное энергосодержащее параболическое ядро, окруженное крыльями с низкой амплитудой. Эти хвосты начинают развиваться на импульсе вблизи точек $t= \pm \tau$, т.е. в области, где отброшенный при теоретическом рассмотрении линейньй член становится важным. Поэтому их описание требует более тщательного анализа формы импульса, которое мы представим в следующем разделе.
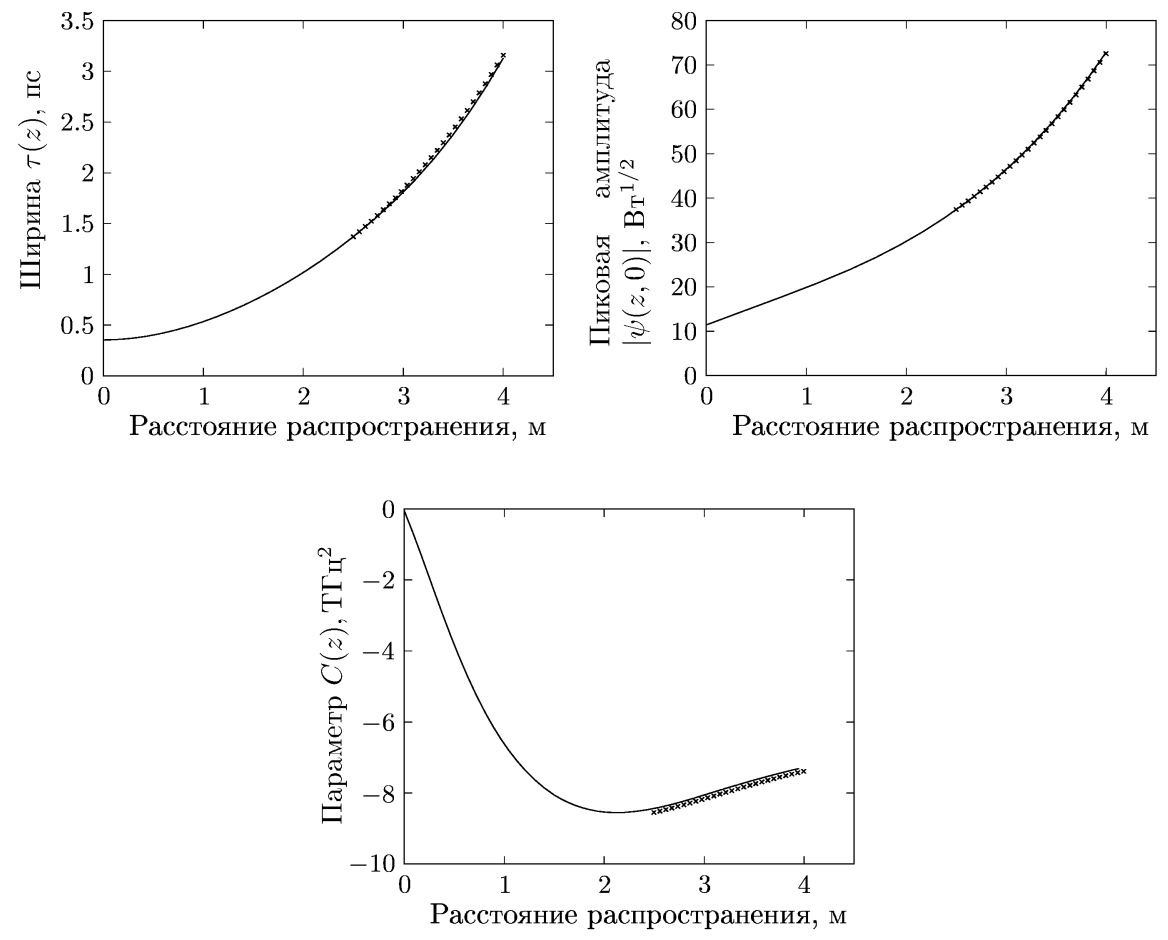

Рис. 2. Эволюция эффективной ширины импульса $\tau(z)$ (вверху слева), пиковой амплитуды $|\psi(z, 0)|$ (вверху справа) и параметра нелинейного изменения частоты $C(z)$ (внизу). Сплошные линии показывают результаты вычислений, а крестики - теоретические предсказания при $z \geqslant 2.5$.м.

Говоря об асимптотическом параболическом режиме, возникающем после некоторого исходного переходного периода, в данном случае после прохождения расстояния 2.5 м, можно количественно сравнить эволюцию параметров импульса, полученную с помощью численного моделирования, с теорией. На рис. 2 сплошными линиями показаны изменения эффективной ширины $\tau(z)$, пиковая амплитуда $|\psi(z, 0)|$ и параметр нелинейно- 


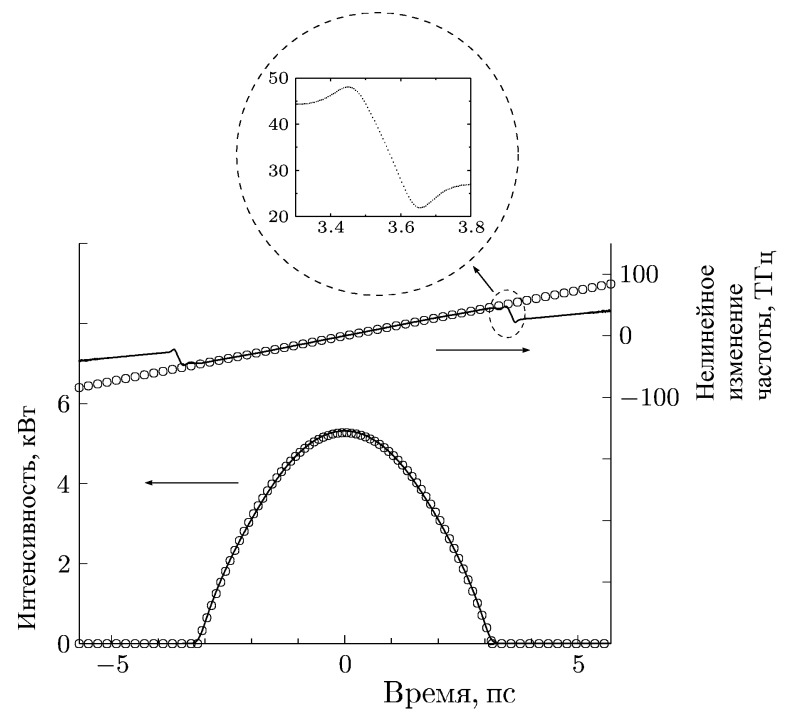

Рис. 3. Интенсивность (левая шкала) и нелинейное изменение частоты (правая шкала) импульса, выходящего из усилителя. Сплошные линии показывают результаты вычислений, а окружности - теоретические предсказания.

го изменения частоты $C(z)$, определенные подгонкой к распространяющемуся импульсу, полученному с помощью численного моделирования. Эти значения хорошо согласуются с ожидаемыми результатами (обозначенными на рисунке крестиками), полученными из решений уравнений $(22),(17)$ и (20) при $z \geqslant 2.5$ м. Кроме того, сплошными кривыми на рис. 3 показаны результаты моделирования для интенсивности и нелинейного изменения частоты (первая производная фазы по времени) импульса, выходящего из усилителя, в сравнении с результатами, получаемыми при решении уравнений (17)-(22) (обозначены окружностями). Эти результаты подтверждают квадратичную зависимость от времени для фазы параболического выходного импульса. Однако мы заметили изменение коэффициента при $t^{2}$ в фазе на "хвостах" параболической части импульса (см. врезку на рис. 3), т.е. там, где нельзя пренебрегать линейным членом в уравнении (1). Это изменение мы учтем в нашем анализе в следуюшем разделе.

\section{4. ЗАДАЧА СШИВКИ}

В предыдушем разделе было показано, что параболическое приближение для формы импульса может описывать центральную часть асимптотически распространяющегося импульса в усиливаюшей среде. Теперь нашей целью является включение в описание хвостов импульса. Для этого мы прежде всего исследовали эволюцию параметра $\epsilon$, определяемого уравнением (11б), на плоскости время-расстояние. На рис. 4 показана эволюция параметра $\epsilon$ как функция расстояния распространения $z$ и нормированного времени $\xi$, вычисленная с помощью численного моделирования уравнения (1). Изменение интенсивности цвета соответствует изменению значений от 0 до 1 , а значения, боль- 
шие 1 , показаны тем же цветом, которьй соответствует 1 . Наверху слева представлена вариация $\epsilon$ на плоскости $(\xi, z)$; наверху справа показана в увеличенном виде более узкая область $\xi$, где ясно видны начальные точки асимптотического режима. На нижнем рисунке показана вариация $\epsilon$ в асимптотическом режиме, начиная с расстояния распространения 2.5 м. Видно, что переход $\epsilon$ от значений, меньших 1 , к значениям, бо́льшим 1 , происходит в узкой области вокруг точек $\xi= \pm 1$, что согласуется с нашими интуитивными ожиданиями. Можно также видеть, что в асимптотическом режиме переходная область (выраженная в самоподобных переменных) не движется с расстоянием.
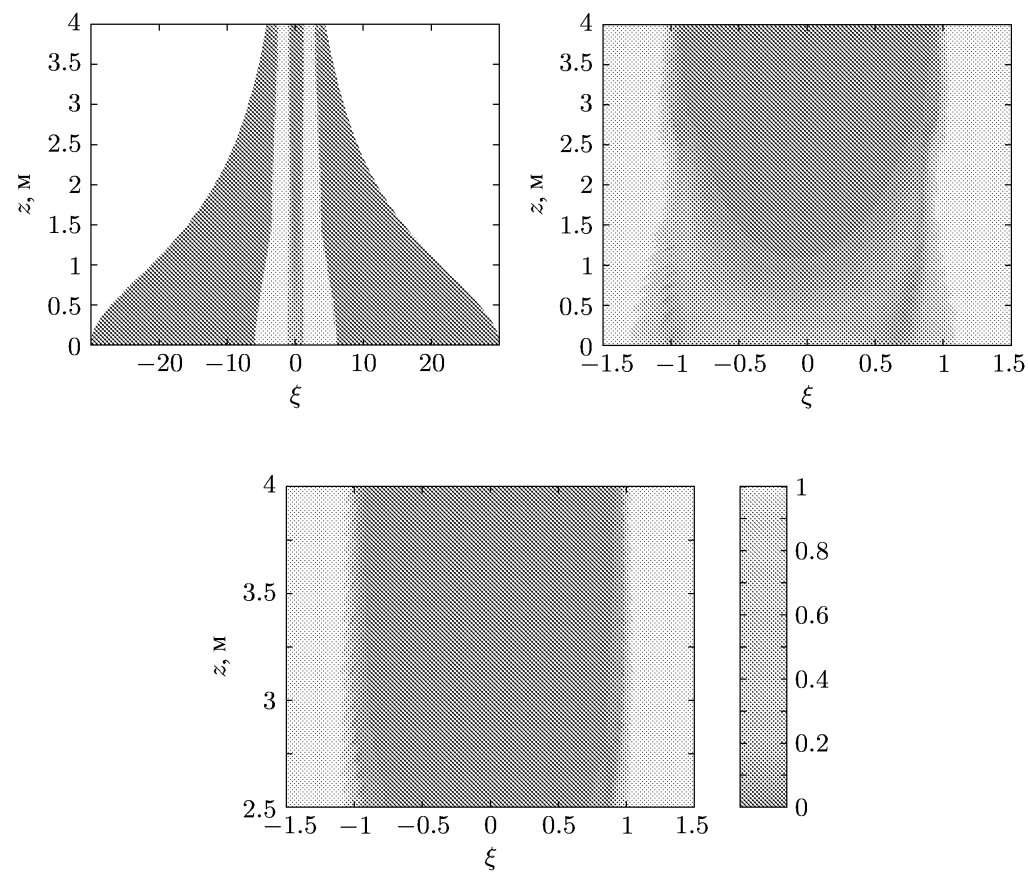

Рис. 4. Эволюция параметра $\epsilon$ как функции расстояния распространения $z$ и нормированного времени $\xi$. Вверху слева - полная плоскость $(\xi, z)$; вверху справа - область $-1.5 \leqslant \xi \leqslant 1.5$; внизу - область $-1.5 \leqslant \xi \leqslant 1.5$ и $z \geqslant 2.5$ м.

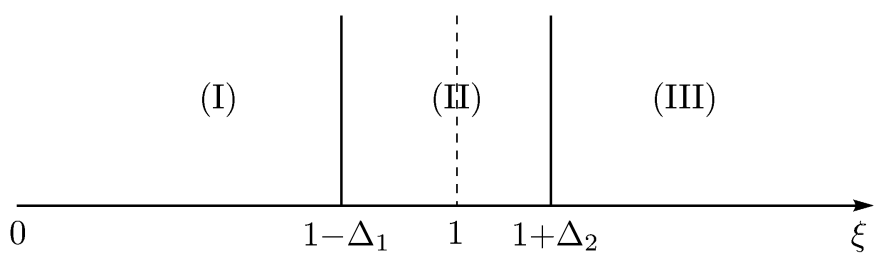

Рис. 5. Схема областей переменной $\xi$, в которых решается уравнение (7). 
На основании этих результатов мы определяем три области на оси $\xi$, как показано на рис. 5. Поскольку задача симметрична по $\xi$, можно ограничиться полуосью $\xi \geqslant 0$. Область I, $0 \leqslant \xi \leqslant 1-\Delta_{1}$, соответствует центральной части асимптотического импульса, где $\epsilon \ll 1$. Область III, $\xi \geqslant 1+\Delta_{2}$, соответствует хвостам импульса, где $\epsilon \gg 1$. Наконец, область II, $1-\Delta_{1} \leqslant \xi \leqslant 1+\Delta_{2}$, является переходной зоной, где $\epsilon \approx 1$. Ее размер параметризован с помощью $\Delta_{1}, \Delta_{2} \ll 1$. Мы вводим два различных $\Delta$-интервала для левой и правой областей вокруг точки $\xi=1$, поскольку решения очевидным образом асимметричны относительно этой точки. В каждой из областей мы строим приближенное решение уравнения (7) или, что эквивалентно, системы (11a) при фиксированном $\eta$ и далее сшиваем решения на гранищах.

В области I рассмотрим квазиклассическое решение, выведенное в разделе 3 , а именHO

$$
\begin{array}{ll}
A=A_{\mathrm{I}}(\xi)=\left[\lambda\left(1-\xi^{2}\right)\right]^{1 / 2}, & 0 \leqslant \xi \leqslant 1-\Delta_{1}, \\
\Phi=\Phi_{\mathrm{I}}(\eta)=\lambda \eta, & 0 \leqslant \xi \leqslant 1-\Delta_{1} .
\end{array}
$$

В области II мы ишем решение в виде

$$
\begin{aligned}
& A=A_{\mathrm{II}}(\eta, \xi), \quad 1-\Delta_{1} \leqslant \xi \leqslant 1+\Delta_{2}, \\
& \Phi=\Phi_{\mathrm{II}}(\eta, \xi)= \begin{cases}\lambda \eta, & \xi=1-\Delta_{1}, \\
\lambda \eta+\Phi_{1}(\eta, \xi), & 1-\Delta_{1}<\xi \leqslant 1+\Delta_{2} .\end{cases}
\end{aligned}
$$

Используя уравнения (24) и предполагая, что $A_{\mathrm{II}}^{2}$ никогда не приближается к нулю, получаем из уравнения (11a)

$$
\begin{gathered}
\frac{\partial \Phi_{1}}{\partial \xi}(\eta, \xi)=\frac{c(\eta)}{2 A_{\mathrm{II}}^{2}(\eta, \xi)} \int_{1-\Delta_{1}}^{\xi} d \xi^{\prime} \frac{\partial A_{\mathrm{II}}^{2}}{\partial \eta}\left(\eta, \xi^{\prime}\right) \\
\Phi_{1}(\eta, \xi)=\frac{c(\eta)}{2} \int_{1-\Delta_{1}}^{\xi} d \xi^{\prime} \frac{1}{A_{\mathrm{II}}^{2}\left(\eta, \xi^{\prime}\right)} \int_{1-\Delta_{1}}^{\xi^{\prime}} d \xi^{\prime \prime} \frac{\partial A_{\mathrm{II}}^{2}}{\partial \eta}\left(\eta, \xi^{\prime \prime}\right) .
\end{gathered}
$$

Подставляя (24) в уравнение (11б), получаем

$$
\frac{1}{c(\eta)} \frac{1}{A_{\mathrm{II}}} \frac{\partial^{2} A_{\mathrm{II}}}{\partial \xi^{2}}-A_{\mathrm{II}}^{2}+\lambda\left(1-\xi^{2}\right)+\frac{\partial \Phi_{1}}{\partial \eta}-\frac{1}{c(\eta)}\left(\frac{\partial \Phi_{1}}{\partial \xi}\right)^{2}=0
$$

Из-за малой вариации $A_{\mathrm{II}}^{2}$ и $c$ при $\eta$, соответствуюшем асимптотическому режиму, из уравнений $(25)$ и (26) легко следует, что $\Phi_{1 \eta}$ и $\left(\Phi_{1 \xi}\right)^{2}$ являются по крайней мере $O\left(\Delta_{1,2}^{2}\right)$. Поэтому, пренебрегая членами порядка не меньше 2 в выражении для $\Delta_{1,2}$, перепишем уравнение (27) в виде [5]

$$
\left[2 \lambda(\xi-1)+A_{\mathrm{II}}^{2}\right] A_{\mathrm{II}}-\frac{1}{c(\eta)} \frac{\partial^{2} A_{\mathrm{II}}}{\partial \xi^{2}}=0 .
$$

С помощью замены переменных

$$
x=(2 \lambda c)^{1 / 3}(\xi-1), \quad \phi=\frac{1}{\sqrt{2}}\left(\frac{c}{4 \lambda^{2}}\right)^{1 / 6} A_{\mathrm{II}}
$$


уравнение (28) можно переписать как специальный случай второго уравнения Пенлеве

$$
\left(x+2 \phi^{2}\right) \phi-\phi_{x x}=0 \text {. }
$$

Граничные условия для уравнения (28) можно сформулировать в терминах асимптотического поведения второго трансцендента Пенлеве. При конкретном выборе свободных параметров асимптотики имеют вид [7]

$$
\begin{aligned}
& \phi \sim\left(-\frac{x}{2}\right)^{1 / 2}, \quad x \rightarrow-\infty, \\
& \phi \sim \frac{1}{2 \sqrt{\pi}} \frac{e^{-2 x^{3 / 2} / 3}}{x^{1 / 4}}, x \rightarrow+\infty .
\end{aligned}
$$

Эти формулы соответствуют следующим граничным условиям для $A_{\mathrm{II}}$ :

$$
\begin{aligned}
& A_{\mathrm{II}}=\left[-\lambda\left(\xi^{2}-1\right)\right]^{1 / 2} \simeq[-2 \lambda(\xi-1)]^{1 / 2}, \quad \xi=1-\Delta_{1}, \\
& A_{\mathrm{II}}=\frac{1}{\sqrt{\pi}}\left(\frac{\lambda}{2 c}\right)^{1 / 4} \frac{e^{-2(2 \lambda c)^{1 / 2}(\xi-1)^{3 / 2} / 3}}{(\xi-1)^{1 / 4}}, \quad \xi=1+\Delta_{2} .
\end{aligned}
$$

Решение граничной задачи, заданной уравнениями $(28),(33),(34)$, дает $A_{\mathrm{II}}(\eta, \xi)$ (при фиксированном $\eta)$. Окончательно, используя $A_{\text {II }}(\eta, \xi)$ в уравнении $(26)$, получаем $\Phi_{\text {II }}(\eta, \xi)$.

В области III мы пренебрегаем нелинейным членом в уравнении (11б) и решаем соответствуюшее линеаризованное уравнение

$$
i F_{\eta}+\lambda \xi^{2} F-\frac{1}{c(\eta)} F_{\xi \xi}=0,
$$

а именно, ишем решение в виде

$$
F(\eta, \xi)=A_{\mathrm{III}}(\eta, \xi) e^{i \Phi_{\mathrm{III}}(\eta, \xi)}=\alpha(\eta) G(\eta, \theta) e^{i R(\eta) \xi^{2}}, \quad \theta=\beta(\eta) \xi, \quad \xi \geqslant 1+\Delta_{2}
$$

Подставляя это выражение в уравнение (35), находим, что

$$
i G_{\eta}-\frac{\beta^{2}(\eta)}{c(\eta)} G_{\theta \theta}=0
$$

выполняется при условиях

$$
\frac{\beta_{\eta}}{\beta}=\frac{4 R}{c}, \quad \frac{\alpha_{\eta}}{\alpha}=\frac{2 R}{c}, \quad R_{\eta}=\frac{4 R^{2}}{c}+\lambda
$$

Из-за граничного условия (34), следуюшего из определения области II, $G$ имеет асимптотику

$$
\begin{aligned}
G(\eta, \theta) \sim & \alpha^{-1}(\eta) \frac{1}{\sqrt{\pi}}\left(\frac{\lambda}{2 c(\eta)}\right)^{1 / 4} \frac{e^{-2(2 \lambda c(\eta))^{1 / 2}(\theta / \beta(\eta)-1)^{3 / 2} / 3}}{\left(\frac{\theta}{\beta(\eta)}-1\right)^{1 / 4}} \times \\
& \times e^{i\left[\Phi_{\mathrm{II}}(\eta, \theta / \beta(\eta))-R(\eta) \theta^{2} / \beta^{2}(\eta)\right]}, \quad \theta \rightarrow \beta(\eta)+0 .
\end{aligned}
$$


Здесь мы применяем условие сшивки фаз $\Phi_{\mathrm{III}}(\eta, \xi)=\Phi_{\mathrm{II}}(\eta, \xi)$ при $\xi \rightarrow 1+0$. Параболическое уравнение (37) с граничным условием (39) решается с помошью формулы двойного потенциала [9]

$$
G(\eta, \theta)=\frac{1}{2 \sqrt{\pi}} \int_{0}^{b(\eta)} d \eta^{\prime} G\left(\eta^{\prime}, \beta(\eta)\left(1+\Delta_{2}\right)\right) \frac{\theta}{\left(b(\eta)-\eta^{\prime}\right)^{3 / 2}} e^{-i \theta^{2} /\left(4\left(b(\eta)-\eta^{\prime}\right)\right)},
$$

где $d b(\eta) / d \eta=\beta^{2}(\eta) / c(\eta)$.

Это завершает построение функции $F(\eta, \xi)$ для всех $\xi$ при фиксированном $\eta$. Условия сшивки требуют подходяшего выбора $\Delta_{1}$ и $\Delta_{2}$. Они должны быть достаточно малыми, чтобы обеспечить сшивку между областями I и II и областями II и III:

$$
|x|=(2 \lambda c(\eta))^{1 / 3}|\xi-1| \gg 1, \quad|\xi-1| \ll 1 .
$$

Таким образом, можно выбрать $\Delta_{1,2}$ из интервала

$$
0<\Delta_{1,2}<(2 \lambda c(\eta))^{-1 / 3+\epsilon}, \quad 0<\epsilon<\frac{1}{3} .
$$

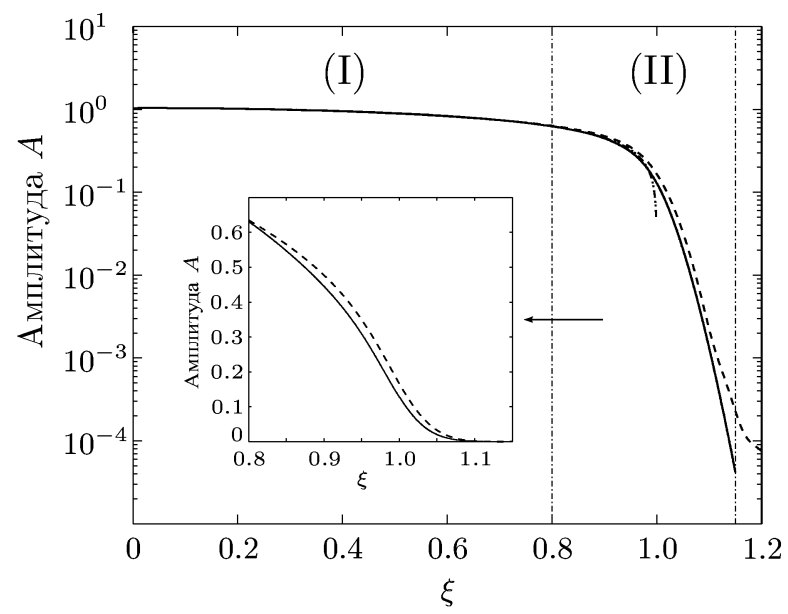

Рис. 6. Амплитуда $A$ как функция от $\xi$ на выходе усилителя. Штриховая линия показывает численное решение, сплошная линия - решение задачи сшивки при $\Delta_{1}=0.2$ и $\Delta_{2}=0.15$, а пунктирная кривая - параболическое решение при $\Delta_{1}=0$. На врезке показаны численное решение и решение задачи сшивки в линейном масштабе.

Мы сравнили решение задачи сшивки с численным решением уравнения (1). Пример представлен на рис. 6 , где показана амплитуда $A$ для $F$ как функция $\xi$ при $0 \leqslant \xi \leqslant 1+\Delta_{2}$ на выходе усилителя. Численное решение можно сравнить с решением задачи сшивки, заданным первым из уравнений (23) и решением уравнения (28). Приведено также параболическое решение первого из уравнений (23) при $\Delta_{1}=0$. Заметим, что параметр $\Delta_{1}$ следует выбрать так, чтобы это параболическое решение первого из уравнений (23) не 
отклонялось от численного в точке $\xi=1-\Delta_{1}$. Пример на рис. 6 соответствует $\Delta_{1}=0.2$ и $\Delta_{2}=0.15$. Можно видеть, что имеется хорошее согласие между аналитическими и численными результатами и различие между ними имеет порядок $\Delta_{1,2}^{2}$, что не противоречит нашим ожиданиям, поскольку члены, которыми мы пренебрегли в уравнении (28), имеют порядок $O\left(\Delta_{1,2}^{2}\right)$. Более точный выбор параметров $\Delta_{1}, \Delta_{2}$ и $\lambda$ должен улучшить точность теоретических результатов.

\section{5. ЗАКЛЮЧЕНИЕ}

Мы исследовали решения НУШ с усилением, которое управляет оптическим импульсом, распространяюшимся в волоконной усиливаюшей среде. Квазиклассические решения с параболическим временни́м профилем в энергосодержащем ядре были аккуратно проанализированы теоретически и численно. Мы представили сшивку параболического решения с линейными низкоамплитудными хвостами импульса. Было показано, что теоретический анализ хорошо воспроизводит решение, полученное путем численного моделирования НУШ.

\section{Список литературы}

[1] G. I. Barenblatt. Scaling, Self-Similarity, and Intermediate Asymptotics. Cambridge: Cambridge Univ. Press, 1996.

[2] V. I. Kruglov, A. C. Peacock, J. M. Dudley, J. D. Harvey. Opt. Lett. 2000. V. 25. P. 1753.

[3] M. E. Fermann, V. I. Kruglov, B. C. Thomsen, J. M. Dudley, J. D. Harvey. Phys. Rev. Lett. 2000. V. 84. P. 6010.

[4] D. Anderson, M. Desaix, M. Karlsson, M. Lisak, M. L. Quiroga-Teixeiro. J. Opt. Soc. Am. B. 1993. V. 10. P. 1185

[5] В. Е. Захаров, Е. А. Кузнецов. ЖЭТФ. 1986. Т. 91. С. 1310.

[6] N. Joshi, M. D. Kruskal. Phys. Lett. A. 1988. V. 130. P. 129; P. A. Clarkson, J. B. McLeod. Arch. Rat. Mech. Anal. 1988. V. 103. P. 97; N. Joshi. Asymptotic studies of the Painlevé equations. In: The Painlevé Property, One Century Later. CRM Series in Mathematical Physics. Ed. R. Conte. New York: Springer-Verlag, 1999. P. 181-227.

[7] A. R. Its, V. Yu. Novokshenov. The Isomonodromic Deformation Method in the Theory of Painlevé Equations. Lecture Notes in Math. V. 1191. Berlin: Springer, 1986; S. P. Hastings, J. B. McLeod. Arch. Rat. Mech. Anal. 1980. V. 73. P. 31.

[8] E. Desurvire. Erbium-Doped Fiber Amplifiers: Principles and Applications. New York: Wiley, 1994.

[9] O. A. Ladyzhenskaja, V. A. Solonnikov, N. N. Ural'ceva. Linear and Quasi-Linear Equations of Parabolic Type. Transl. of Math. Monographs. V. 23. Providence, RI: AMS, 1968. 